\title{
Evidence that single-stranded DNA breaks are a normal feature of koala sperm chromatin, while double-stranded DNA breaks are indicative of DNA damage
}

\author{
Yeng Peng Zee, Carmen López-Fernández ${ }^{1}$, F Arroyo ${ }^{1}$, Stephen D Johnston, William V Holt ${ }^{2}$ \\ and Jaime Gosalvez ${ }^{1}$ \\ The University of Queensland, Gatton, Queensland 4343, Australia, ${ }^{1}$ Universidad Autónoma de Madrid, 20849 \\ Madrid, Spain and ${ }^{2}$ Institute of Zoology, Zoological Society of London, Regent's Park, London NW1 4RY, UK
}

Correspondence should be addressed to WV Holt; Email: bill.holt@ioz.ac.uk

\begin{abstract}
In this study, we have used single and double comet assays to differentiate between single- and double-stranded DNA damage in an effort to refine the interpretation of DNA damage in mature koala spermatozoa. We have also investigated the likelihood that single-stranded DNA breakage is part of the natural spermiogenic process in koalas, where its function would be the generation of structural bends in the DNA molecule so that appropriate packaging and compaction can occur. Koala spermatozoa were examined using the sperm chromatin dispersion test (SCDt) and comet assays to investigate non-orthodox double-stranded DNA. Comet assays were conducted under 1) neutral conditions; and 2) neutral followed by alkaline conditions (double comet assay); the latter technique enabled simultaneous visualisation of both single-stranded and double-stranded DNA breaks. Following the SCDt, there was a continuum of nuclear morphotypes, ranging from no apparent DNA fragmentation to those with highly dispersed and degraded chromatin. Dispersion morphotypes were mirrored by a similar diversity of comet morphologies that could be further differentiated using the double comet assay. The majority of koala spermatozoa had nuclei with DNA abasic-like residues that produced single-tailed comets following the double comet assay. The ubiquity of these residues suggests that constitutive alkali-labile sites are part of the structural configuration of the koala sperm nucleus. Spermatozoa with 'true' DNA fragmentation exhibited a continuum of comet morphologies, ranging from a more severe form of alkaline-susceptible DNA with a diffuse single tail to nuclei that exhibited both single- and double-stranded breaks with two comet tails.

Reproduction (2009) 138 267-278
\end{abstract}

\section{Introduction}

Prior to spermiogenesis, the spermatid nucleus displays the typical nucleosomal chromatin pattern seen in somatic cells. As spermiogenesis progresses, the DNA molecule undergoes a series of mechanical and biochemical changes that allows the chromatin to ultimately pack tightly into a highly compact form. Nuclear condensation is facilitated by the progressive replacement of histones with protamines during mid-spermiogenesis. As this occurs, torsional stress is generated in unconstrained supercoils of DNA; in order to ease these tensional forces and facilitate protamine deposition, the DNA molecule must undergo a series of programmed DNA breaks and subsequent repairs (Boissonneault 2002, Marcon \& Boissonneault 2004). Evidence of such activity has been found in the DNA of elongating mouse spermatids and human round spermatids, where it is likely to be mediated by topoisomerase II (McPherson \& Longo 1993, Marcon \& Boissonneault 2004).
Occasionally, normal sperm DNA packaging is compromised by a range of factors resulting in detrimental effects upon the configuration and function of the ultimate DNA molecule. Some external factors such as environmental toxicants and drugs compromise the quality of DNA during spermiogenesis itself, rendering the resultant spermatozoa subfertile or unable to support embryo development after fertilisation, while other external factors or treatments, possibly including cryopreservation, impact directly upon the mature spermatozoa, thereby inducing DNA damage more directly. Within this concept, we include 'sperm DNA fragmentation' or 'sperm DNA damage', which is regarded as a detrimental change in the topology of the chromatin complex. Despite the fact that genomic integrity is vital to the achievement of normal embryonic development and pregnancy, sperm DNA quality is currently not routinely assessed as part of the standard male fertility assessment in many species. Nevertheless, sperm DNA fragmentation is progressively being 
demonstrated as a highly predictive indicator of male factor infertility (Agarwal \& Said 2003), particularly when accounting for early embryonic loss, which cannot always be explained by traditional parameters of sperm quality (Zini et al. 2001, Evenson et al. 2002).

To understand the significance of DNA fragmentation in spermatozoa, it is first essential to differentiate between DNA modifications that are induced as a normal part of the packaging process and those that are abnormal and undesirable. The results of previous investigations into DNA fragmentation of the koala sperm nucleus (Johnston et al. 2007), initially undertaken to understand the causes of cryoinjury in these spermatozoa, led us to propose a model of sperm chromatin organisation involving specific regions of DNA, which exhibit a high sensitivity to protein depletion and may be especially well adapted to molecular bonding in order to achieve maximum chromatin compaction. Here, we investigate this model in more detail, specifically asking whether these particular DNA domains contain unpaired singlestranded DNA motifs or even apurinic or apyrimidinic regions. If so, these regions, which render the DNA molecule highly sensitive to protein removal or to alkaline treatments, would be unusually liable to produce single-stranded DNA stretches. These nonorthodox DNA configurations are likely to be an important factor in the packaging of the DNA in species such as the koala where disulphide bonds are absent in the chromatin. However, as they are also liable to being easily altered, the existence of these DNA moieties distributed throughout the genome might help to explain the occurrence of increased chromatin 'relaxation' following cryopreservation.

The sperm chromatin dispersion test (SCDt) is a simple microgel slide-based assay that has been used to assess DNA fragmentation in spermatozoa in a wide range of species. Recently, our research has focussed on modifying and adapting the SCDt, originally developed for human spermatozoa (Fernandez et al. 2003), to the unique chromatin structure of a range of domestic and wild animals (Gosálvez et al. 2006). Particular attention has been given to koala spermatozoa, because in this species, as is the case for the majority of marsupials, the protamines are devoid of cysteine residues, rendering it impossible to form highly stable protein configurations through disulphide bonds (Fifis et al. 1990, TempleSmith 1994). In the original Halosperm technique, developed for humans, the method involves treating spermatozoa with an acidic solution to denature DNA prior to a lysing procedure that removes proteins and membranes (Fernandez et al. 2003). With this technique, spermatozoa with a halo of dispersed DNA have nonfragmented DNA. In a modified version of this technique, which was developed for boar spermatozoa, the acid-treatment procedure was removed (Enciso et al. 2006). In this variant of the original technique, two primary morphological sperm types are observed after controlled protein depletion: 1) spermatozoa with a large halo of dispersed chromatin surrounding a variable-sized nuclear core, which corresponds to those containing fragmented DNA; and 2) spermatozoa not exhibiting a halo of chromatin dispersion surrounding a nuclear core; these are identified as spermatozoa with intact DNA integrity. Koala spermatozoa also exhibit these two distinctive nuclear morphotypes following the SCD procedure (Johnston et al. 2007), but their interpretation is not straightforward. The nonfragmented nuclei can be subdivided into morphotypes 1 (no halo) and 2 (small compact halo) and those with a distinctive 'stellar' morphology of dispersed fragmented chromatin (morphotype 3); morphotype 3 has been proposed as representing 'true' DNA fragmentation, where both single- and double-strand breaks (SSB and DSB) are present.

In an attempt to support the suggested model of chromatin packaging in the koala sperm nucleus, the present study aimed to investigate the occurrence of DNA configurations other than the orthodox doublestranded DNA helix. These experiments were conducted to gain information about the nature of the DNA damage in spermatozoa identified with the SCD technique as having fragmented DNA. We propose the hypothesis that koala sperm morphotypes 1 and 2 mainly contain DNA sequences that are highly sensitive to gentle alkaline environments due to the presence of unpaired singlestranded DNA motifs or the presence of apurinic or apirimidinic sites that are part of constitutive structural DNA modifications.

\section{Results \\ Experimental designs}

The initial study on validation of the SCDt for koala spermatozoa revealed the presence of three sperm nuclear morphotypes, KM1, KM2 and KM3, where KM3 was regarded as representing sperm nuclei with fragmented DNA (Johnston et al. 2007). Since this original study, we have subsequently become aware that these sperm nuclear morphotypes may not be as discrete as first thought, but form parts of a continuum of DNA fragmentation. The first experiment in this study was, therefore, to document the range of SCDt morphotypes found in koala spermatozoa more comprehensively. For this purpose, we focused on two koalas out of a larger cohort of 22 animals; basic pre-freeze and post-thaw semen parameters for these two koalas (K5 and K7) were similar to those of the other 20 (Table 1), but they represented animals that either showed a consistently high (K5) or low (K7) level of DNA damage when this was assessed dynamically over a 24-h period. Frozen spermatozoa from $\mathrm{K} 5$ and $\mathrm{K} 7$ were thawed at $35^{\circ} \mathrm{C}$ for $1 \mathrm{~min}$ and sperm DNA fragmentation index 
Table 1 Comparison of semen parameters for koalas $\mathrm{K} 5$ and $\mathrm{K} 7$ with the remainder of the koalas examined in this study.

\begin{tabular}{|c|c|c|c|c|c|c|c|}
\hline & $N$ & Mean $(\%)$ & Median (\%) & Lower quartile & Upper quartile & Koala K5 (\%) & Koala K7 (\%) \\
\hline \multicolumn{8}{|l|}{ Before freezing } \\
\hline Motility & 20 & 78.1 & 81.0 & 71.0 & 89.5 & 90 & 82 \\
\hline LHMMP & 20 & 68.1 & 76.0 & 50.5 & 91.5 & 87 & 93 \\
\hline LLMMP & 20 & 21.2 & 13.0 & 2.0 & 38.0 & 7 & 1 \\
\hline Chromatin relaxation & 20 & 4.8 & 2.0 & 1.0 & 6.0 & 2 & 0 \\
\hline \multicolumn{8}{|l|}{ After thawing } \\
\hline Motility & 20 & 45.2 & 50 & 31.5 & 55.0 & 55 & 3 \\
\hline LHMMP & 20 & 40.1 & 41.5 & 25.0 & 58.0 & 55 & 47 \\
\hline LLMMP & 20 & 12.4 & 10.5 & 6.0 & 14.5 & 3 & 15 \\
\hline Chromatin relaxation & 20 & 34.2 & 31.0 & 23.0 & 47.0 & 53 & 40 \\
\hline $\operatorname{sDFI}(T=0)$ & 12 & 6.8 & 4.7 & 3.33 & 9.33 & 0 & 2.7 \\
\hline
\end{tabular}

Koala K7 and K5 were omitted for the purposes of calculating summary statistics.

(sDFI) was evaluated using the SCDt immediately (T0) and after $24 \mathrm{~h}$ incubation at $35{ }^{\circ} \mathrm{C}$ (T24). In experiments 2 and 3 , these same semen samples were also processed through a neutral comet and a double (neutral + alkaline) comet assay respectively, so that all three evaluations of sperm DNA damage could be directly compared. The neutral comet and the double comet assays produced a range of comet morphologies, which were subsequently interpreted with respect to their potential composition of double- and single-stranded DNA breaks.

\section{Experiment 1: koala sperm morphology and the incidence of $s D F I$ following the SCDt}

Figure 1 shows a selection of the range of sperm nuclear morphologies from koalas 5 and 7 following the SCDt at T0 and T24. Koala spermatozoa morphology interpreted as showing non-fragmented DNA had no halo or had a small and compact halo of DNA (Fig. 1a), whereas nuclei with fragmented DNA exhibited a highly dispersed distribution of DNA fragments (Fig. 1b-f). It is clear from Fig. $1 b-f$ that the dispersion of DNA fragments for the koala sperm nucleus after the SCDt can be expressed as part of a dynamic or continuous process. There was a corresponding increase in the size of the DNA halo as the nuclear core reduced in size. This continued to the point where the nucleus was only a remnant and the fragments of the chromatin were so dispersed that they were not observed in the immediate vicinity of the remaining components (flagellum) of the spermatozoon (Fig. 1f). As the chromatin dispersed, the halo became less apparent, and it was difficult to visualise even with the use of a highly specific DNA-binding fluorescent probe such as GelRed. The nuclear core also became progressively smaller (compare Fig. 1a with f). Electronic image filtering was used to enhance these differences and the results are depicted in Fig. $1 a^{\prime}-f^{\prime}$.

The incidence of non-fragmented (Fig. 1a), fragmented (Fig. 1b-e) and degraded (Fig. 1f) sperm nuclei for $\mathrm{K} 5$ and $\mathrm{K} 7$ is reported in Table 2. These data indicate that $\mathrm{K} 5$ had a higher incidence of fragmented DNA
(sDFI $=$ fragmented + degraded) at T0 and T24 compared with that of $\mathrm{K} 7$, and that sperm DNA fragmentation in both animals increased following incubation at $35^{\circ} \mathrm{C}$ for $24 \mathrm{~h}$.

\section{Experiment 2: comet morphology and the incidence of sperm DNA fragmentation following a neutral comet assay}

The typical appearance of a range of comets following application of the neutral comet assay is shown in Fig. 2, where spermatozoa with various comet tail lengths and densities were observed. Following treatment with the lysing solution used for the SCDt, the neutral comet assay was able to differentiate between different degrees of severity in koala sperm DNA damage. While sperm nuclei displaying a compact comet head and no comet tail (Fig. 2a) represented non-fragmented DNA, spermatozoa with fragmented DNA exhibited varying levels of changes to the nuclear core and comet tail length, which could be visualised as a continuum (Fig. 2b-i). The comet tail lengths of some koala sperm nuclei with damaged DNA increased while the size of the nuclear core remained relatively constant (Fig. 2b-d). Other spermatozoa, presumably with a much higher degree of DNA damage, showed a reduction in the size of the nuclear core and a decrease in the presence of DNA fragments in the immediate proximity of the nuclear core of the comet tail, as the DNA fragments in the comet progressively degraded and dispersed. The DNA of degraded sperm nuclei had presumably migrated far away from the nuclear core resulting in one that is extremely reduced in size (Fig. $2 \mathrm{f}-\mathrm{i}$ ).

Densitometric analysis (Fig. 2e and j) of the comets (Fig. $2 a^{\prime}-d^{\prime}$ and $f^{\prime}-i^{\prime}$ ) provided a means of quantifying and confirming the patterns observed by visual assessment (Fig. 2a-i). An interesting feature of the analysis for the type of comets shown in Fig. 2a-d was that the intensity of the fluorescence in the comet (pixel values along the comet) was similar among different spermatozoa; this indicates that the amount of DNA in the 

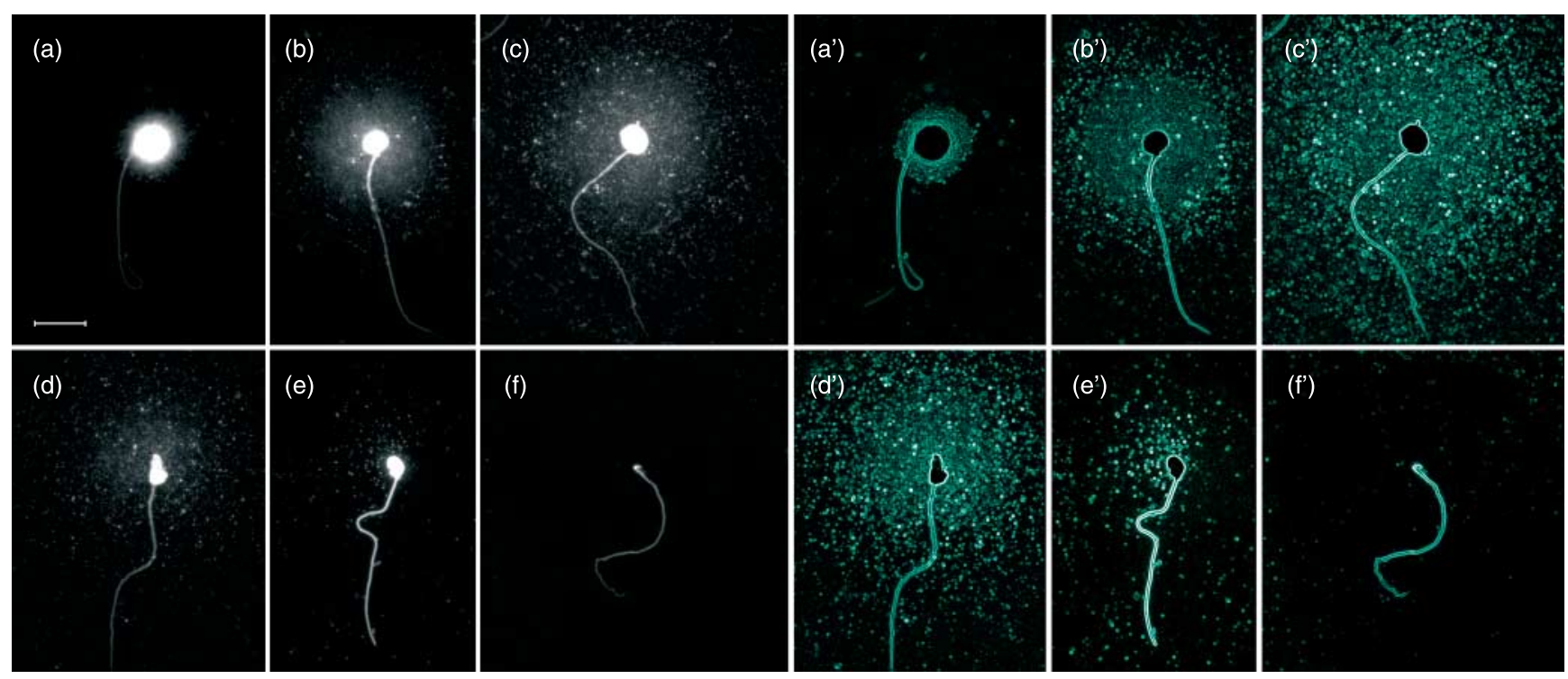

Figure 1 Koala sperm nuclear morphology following SCDt. (a) Non-fragmented DNA of morphotype 1 or 2; (b-f) various degrees of DNA fragmentation of morphotype $3 ; \mathrm{a}^{\prime}-\mathrm{f}^{\prime}$ are digitally enhanced images to highlight the distribution and intensity of chromatin dispersal.

respective comet heads was also similar. The mean ( \pm s.D.) pixel of 20 sperm nuclei was $47 \pm 22$ (Fig. 2e). However, the length of the comet tail varied from one spermatozoon to another (Fig. 2e), with the size of the comet tail ranging from 100 to 550 pixels (see colour code in Fig. 2e to mark the position of the more distant migrant DNA fragment). When the densitometric analysis was performed on the highly fragmented sperm nuclei (Fig. $2 \mathrm{f}^{\prime}-\mathrm{i}^{\prime}$ ), there was considerable variability, both in terms of the size of the nuclear core

Table 2 Comparative analysis of sperm DNA fragmentation index (SDFI) in koala 5 and koala 7 immediately upon thawing (T0) and after $24 \mathrm{~h}$ incubation at $35^{\circ} \mathrm{C}(\mathrm{T} 24)$ using three different methods of sDFI assessment.

\begin{tabular}{lrrrrr}
\hline & \multicolumn{2}{c}{ Koala 5 } & & \multicolumn{2}{c}{ Koala 7 } \\
\cline { 2 - 3 } \cline { 5 - 6 } & \multicolumn{1}{c}{ T0 } & T24 & & T0 & T24 \\
\hline SCD & & & & & \\
Not fragmented & 84.7 & 32.7 & & 93.6 & 84.6 \\
Fragmented & 15.3 & 64.7 & & 6.3 & 15.0 \\
Degraded & 0.0 & 2.7 & & 0.0 & 0.3 \\
sDFI & 15.3 & 67.3 & & 6.4 & 15.3 \\
Neutral comet & & & & & \\
Not fragmented & 82.0 & 44.0 & & 92.3 & 84.0 \\
Fragmented & 18.0 & 50.7 & & 0.0 & 0.0 \\
Degraded & 0.0 & 5.3 & & 7.6 & 16.0 \\
sDFI & 18.0 & 56.0 & & 7.6 & 16.0 \\
Neutral+alkaline comet & & & & & \\
Structural & 76.7 & 33.0 & & 85.0 & 81.3 \\
SSB & 9.7 & 32.3 & & 7.3 & 15.0 \\
DSB + SSB & 6.0 & 1.0 & & 0.0 & 0.0 \\
Degraded & 7.7 & 33.7 & & 0.0 & 3.6 \\
sDFI & 23.3 & 67.0 & & 7.3 & 18.6 \\
& & & &
\end{tabular}

SSB, single-strand break; DSB, double-strand break. and the length of the comet tail (see Fig. 2j). The nuclear cores of the spermatozoa were substantially smaller in Fig. $2 \mathrm{f}-\mathrm{i}$ and there was an accompanying progressive reduction in the length of the comet tail as the DNA fragments became more dispersed.

The primary comet morphologies following the neutral assay are schematically illustrated in the first column of Fig. 3; these include fragmented (Fig. 3a) and degraded (Fig. 3b) DNA. The most degraded sperm DNA comets have a small nuclear core and a small halo without any visible comet tail (Fig. 3 $b^{\prime}$ ). The incidences of nonfragmented, fragmented and degraded sperm nuclei in $\mathrm{K} 5$ and $\mathrm{K} 7$ following the neutral comet assay are reported in Table 2. The sDFI (fragmented+degraded) of K5 was greater than $\mathrm{K} 7$ at T0 and even more so after $24 \mathrm{~h}$ incubation of the spermatozoa at $35^{\circ} \mathrm{C}$.

\section{Experiment 3: comet morphology and the incidence of sperm DNA fragmentation following a double (neutral+alkaline) comet assay}

Following the double comet assay, four main groups of spermatozoa were observed (schematically represented in Fig. 3c-f). The first of these initially showed no evidence of DNA migration following the first electrophoresis under neutral conditions, but when subsequently processed under alkaline conditions resulted in comets that were indicative of SSB (Fig. 3c and d). Comets represented by Fig. 3c had a consistent nuclear core and comet tail size (Fig. 4a-d). Note that the direction of the electrophoresis migration under the alkaline condition was perpendicular to that resulting from electrophoresis under the neutral condition 

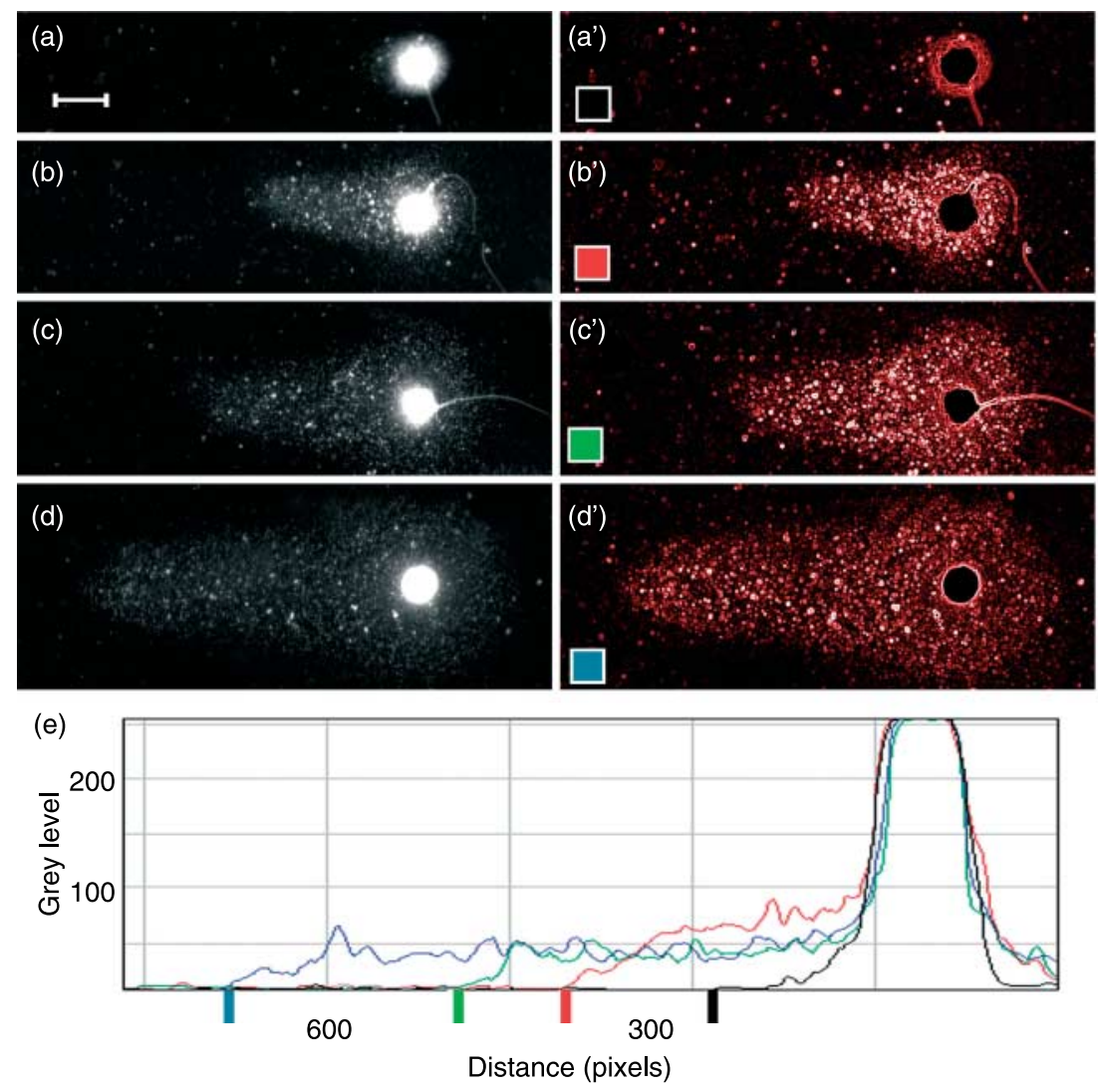
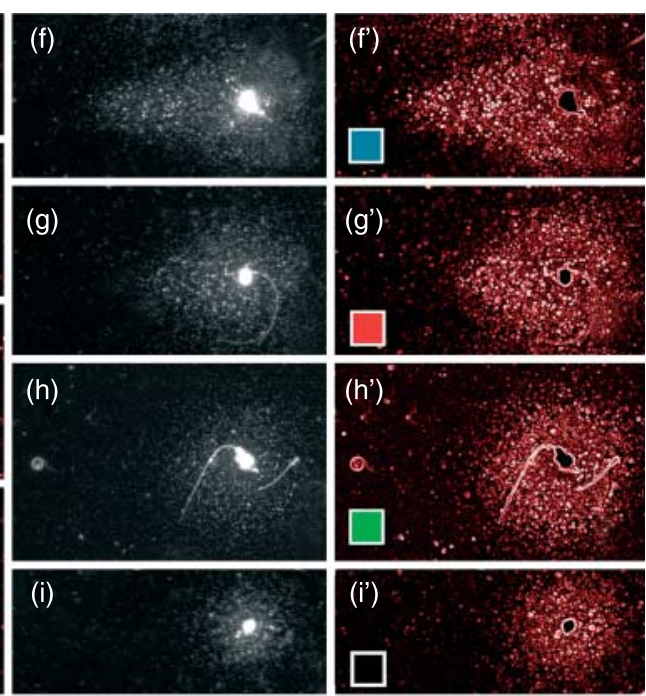

(j)

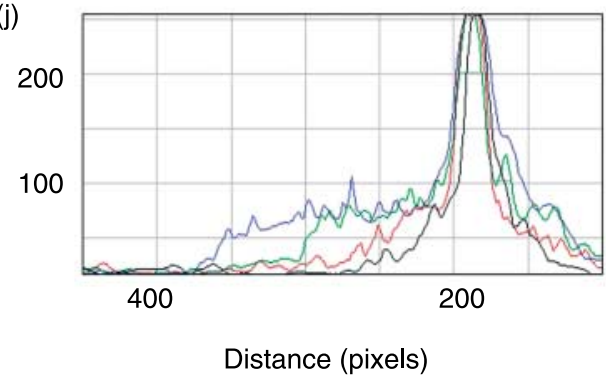

Figure 2 Typical appearance of comets following application of the neutral comet assay. (a) Non-fragmented koala sperm nucleus; (b-d) fragmented koala sperm nuclei with a constant size nuclear core but varying tail lengths; (e) densitometric representation of the four nuclei in figures b-d; $(\mathrm{f}-\mathrm{i})$ grossly fragmented koala sperm nuclei with a reduced nuclear core and varying comet tail lengths; (j) densitometric representation of the four nuclei in figures $\mathrm{f}-\mathrm{i}$. In the highly fragmented nuclei, the comet tail and core are smaller because the amount of chromatin recovered after lysis is also low - compare Fig. $2 \mathrm{f}$ with $\mathrm{i}$ against Fig. $1 \mathrm{~d}$ with $\mathrm{f}$.

(compare diagrams in Fig. 3a and c-e). In contrast to the neutral comet assay, where comet tail lengths varied according to the level of DNA damage, the lengths of migrant DNA stretches resulting from the alkaline comet assay were similar in all the cases (Fig. 4a-d) and moved in the order of 300 pixels of magnitude (Fig. 4e). While the most distant chromatin fragments appeared to migrate the same distance on the microgel, there was, however, significant variation in chromatin density in each of the comet tails (Fig. 4a-d and g). Given that all sperm nuclei showed evidence of some form of SSB, this type of DNA morphology was categorised as a constitutional structural feature of koala sperm DNA.

The other three groups of koala sperm DNA comet morphologies are likely to be indicative of true DNA damage. The first of these is represented in Fig. 4f, which was characterised by DNA fragments migrating in the same direction as those described above, but had longer comet tails and a smaller nuclear core. Interestingly, in this type of comet, there were no traces of perpendicular migration of DNA fragments and the size of the tail could be two times or more than that observed in the structural type described above. A schematic illustration of this type of comet is shown in Fig. $3 \mathrm{~d}$ and is labelled as SSB. This type of comet is likely to contain more singlestranded DNA breaks than that found in the structural type. Another group of comets representing fragmented DNA consisted of DNA migration in two directions. Figure 5a-d illustrate a range of koala spermatozoa exhibiting comets with two tails, which are perpendicular to each other and which signifies the presence of both DSB (horizontal direction) and SSB (vertical direction) showing different extent of DNA damage for SSB and DSB; Fig. 3e is a schematic representation of these types of comets. The final type of comet observed had highly degraded nuclear cores, where the DNA had dispersed to the point where only a small remnant of the nuclear core was present. The images of these spermatozoa are similar to those included in Fig. $1 \mathrm{f}$ and a diagram is shown in Fig. $3 \mathrm{f}$.

The incidence of structural, SSB, SSB +DSB and degraded sperm nuclei in $\mathrm{K} 5$ and $\mathrm{K} 7$ following the neutral and double comet assays is reported in Table 2. The sDFI (SSB + DSB + degraded) of K5 was greater than $\mathrm{K} 7 \mathrm{at} \mathrm{T} 0$ and even more so following $24 \mathrm{~h}$ incubation of the frozen-thawed spermatozoa at $35^{\circ} \mathrm{C}$. 


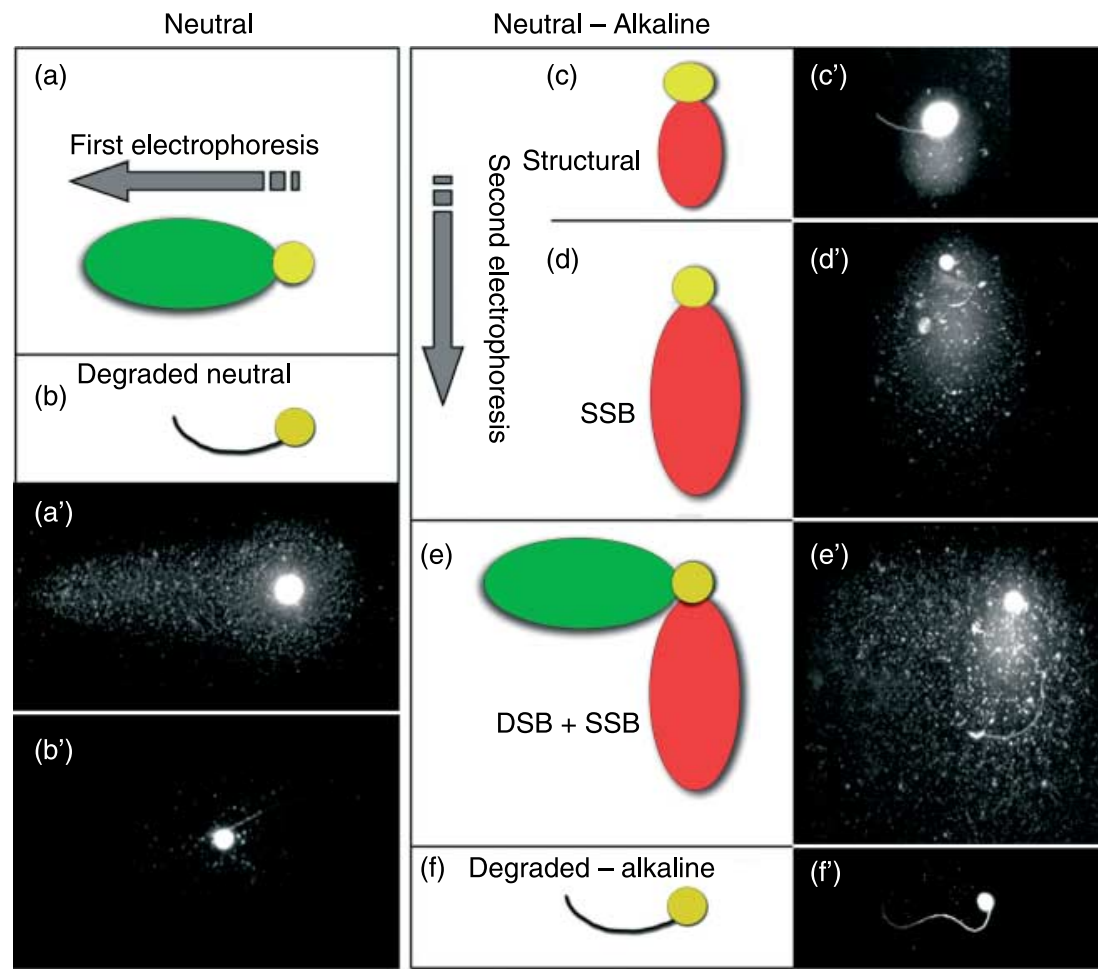

Figure 3 Schematic representations and the corresponding micrographs of koala sperm comets following neutral and neutral + alkaline comet assays. (a) Spermatozoon with single comet tail following neutral comet assay; (b) spermatozoon with degraded nucleus following neutral comet assay; ( $a^{\prime}$ and $\left.b^{\prime}\right)$ micrographs of (a) and (b) respectively. (c and $d$ ) Sperm nuclei after the double comet assay, where DNA migration was not detected after the first electrophoresis under neutral condition, but only after the second electrophoresis under alkaline condition; $\left(c^{\prime}\right)$ and $\left(d^{\prime}\right)$ micrographs of $(c)$ and (d) respectively. (e and $\mathrm{e}^{\prime}$ ) Schematic representation and micrograph respectively of koala spermatozoon with two comet tails; the horizontal DNA migration following the first electrophoresis under neutral condition represents DSB and the vertical DNA migration following the second electrophoresis under alkaline condition represents SSB.

( $f$ and $f^{\prime}$ ) Schematic representation and micrograph respectively of degraded sperm nucleus following the double comet assay; spermatozoa classified as degraded have only a small remnant of nuclear core without any sings of comet head or comet tail.
Comparative analysis of sDFI measured by SCDt, neutral comet and double (neutral + alkaline) comet assays

The incidence of sperm DNA fragmentation in $\mathrm{K} 5$ and $\mathrm{K} 7$ following a comparative analysis using the SCDt, neutral comet assay and double (neutral + alkaline) comet assay is shown in Table 2 and summarised in Fig. 6. Spermatozoa were analysed immediately upon thawing (T0) and after $24 \mathrm{~h}$ incubation at $35^{\circ} \mathrm{C}$ (T24). When a comparative analysis of sDFI of each koala was performed using three different methods of sDFI assessment (SCDt, neutral and double comet assays), the rate of sperm DNA fragmentation was essentially the same. There was, however, a difference in the rate of sDFI between animals at T0 and even more so after $24 \mathrm{~h}$ of sperm incubation at $35^{\circ} \mathrm{C}$. The capacity to detect total DNA damage was higher when the double comet assay was used (blue lines in Fig. 6).

\section{Discussion}

The results of the present study revealed good agreement between the SCDt, the neutral comet assay and neutral + alkaline comet assay (Table 2). The SCDtassessed sDFI reflected the combined incidence of spermatozoa with degraded DNA and those showing substantial occurrence of DNA that was highly susceptible to alkaline denaturation assessed using the comet assays. We therefore propose that koala sperm nuclei with degraded DNA and those with a high incidence of alkaline-susceptible DNA can be collectively considered as representing spermatozoa with true
DNA fragmentation. Furthermore, the incidence of spermatozoa with degraded nuclei and those exhibiting large haloes of chromatin dispersion reflected the proportion of spermatozoa detected by the neutral comet assay as having DSB.

These results are strong evidence that DNA fragmentation in koala spermatozoa is associated with DSB and that the proportion of these spermatozoa in the ejaculate is an indication of the true DNA fragmentation index. The presence of comets produced under alkaline conditions in this study demonstrated that DNA motifs, alternative to the standard continuous double-stranded DNA molecule, generated single-stranded DNA stretches and occurred uniformly in all spermatozoa; these may therefore be a structural feature of the normal koala sperm nucleus. In addition to these two DNA configurations, another category of spermatozoa was observed; these possessed 'non-structural' SSB that produced comets with tails that were larger than those containing only structural SSB.

A typical koala semen sample analysed for sperm DNA fragmentation will therefore contain extensive SSB, which are likely to be a structural feature of the nucleus, and DSB in varying albeit relatively low proportions in each individual but which would be considered true DNA damage. In the present study, we observed that the SCD technique mainly revealed the presence of DSB or KSM-3 (Johnston et al. 2007). All koala spermatozoa exhibited SSB after alkaline treatment from the double comet assay; however, these structural SSB were not clearly differentiated by the koala SCDt. For example, 

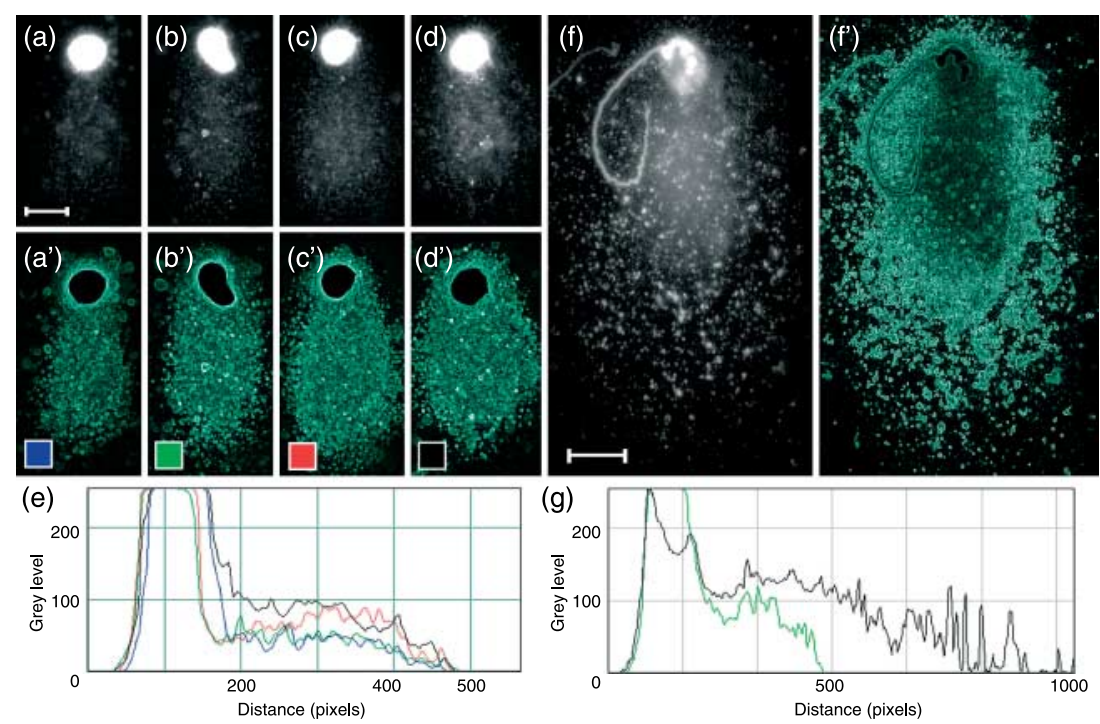

Figure 4 Sperm comets with single tails following application of the double comet assay. (a-d) Koala sperm nuclei showing similarly sized nuclear cores and similar sized tails; (e) densitometric analysis of figures $a-d$; (f) massive presence of single-stranded DNA breakage with a reduced nuclear core and long comet tail; (j) densitometric representation of figure $(f)$ to compare with the average (green line) in structural comets. the SCDt in a previous study (Johnston et al. 2007) identified KSM-1 and KSM-2 as sperm nuclei with compact nuclear cores with no or only small halos, but gave no indication of the structural SSB inherent in the chromatin.

Conceptually, the comet assay performed under nondenaturing conditions detects DSB, although SSB may also contribute partly to the migration due to relaxation of the chromatin supercoils caused by the strand breaks (Ostling \& Johanson 1984). However, the alkaline comet assay may be problematic since native intact DNA from human spermatozoa is strongly sensitive to alkaline denaturation, possibly as a result of a specific chromatin-DNA conformation (Singh et al. 1989, Muriel et al. 2006). A variant of the SCDt using only the lysing solution without previous acid unwinding treatment preferentially detects DSB; this corresponds to a nondenaturing, diffusion-like assay, where massive DSB are discernable as wide propagation of the DNA fragments from the residual nuclear core of the sperm head (Tice et al. 2000, Enciso et al. 2006). Nevertheless, strong correlations in sperm DNA fragmentation assessment have always been obtained between electrophoresis, enzymatic and denaturing detection systems, at least with ejaculated spermatozoa (Gorczyca et al. 1993, Aravindan et al. 1997, Chohan et al. 2006).

While an earlier study identified three predominant koala sperm nuclear morphotypes with the use of SCDt (Johnston et al. 2007), the current study was conducted across a larger number of koalas (YP Zee, C López-Fernández, F Arroyo, SD Johnston, WV Holt \& J Gosalvez 2008, unpublished observations) so that it quickly became evident that it was possible to identify a range or continuum of nuclear morphotypes, from those displaying no evidence of DNA fragmentation to those with highly dispersed and degraded chromatin. This range of SCDt morphotypes has now been documented in the current study and supersedes the descriptors previously provided in Johnston et al. (2007).

An unexpected finding in this study was the possible presence of extensive alkali-labile sites (ALS), which appeared to produce comet tails of a consistent size after alkaline treatment. In the context of using koala spermatozoa for artificial reproduction, this phenomenon is of great interest. Incubation of koala spermatozoa following cryopreservation results in sperm nuclei with a high incidence of 'chromatin swelling' (Johnston et al. 2006, Zee et al. 2008) or 'chromatin
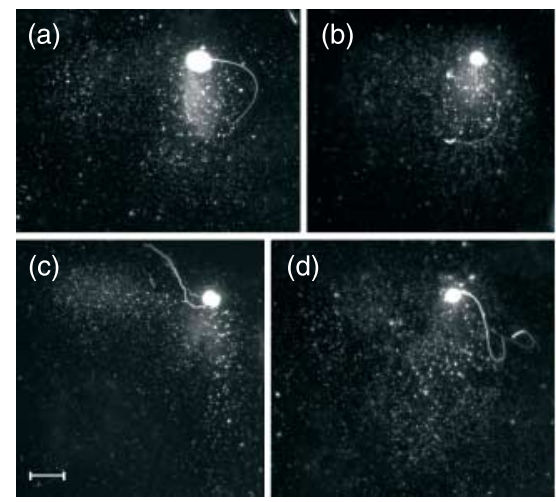
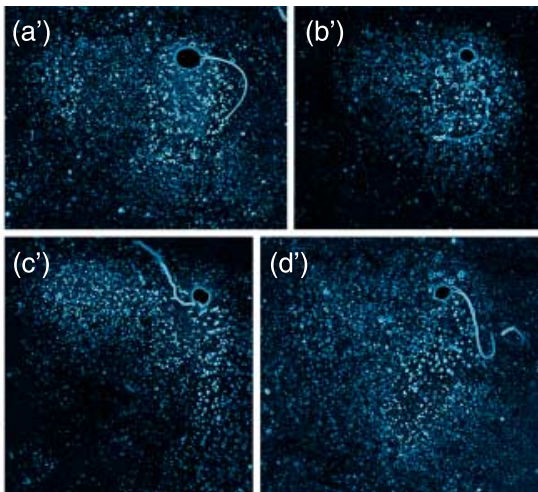

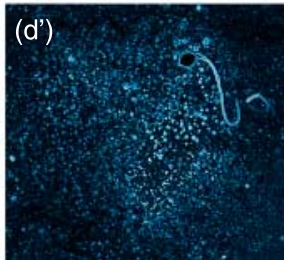

Figure 5 Koala sperm comets with two tails following application of the double comet assay. There are differences in the length of the tail and the DNA density in the tail varied between individual spermatozoa. 


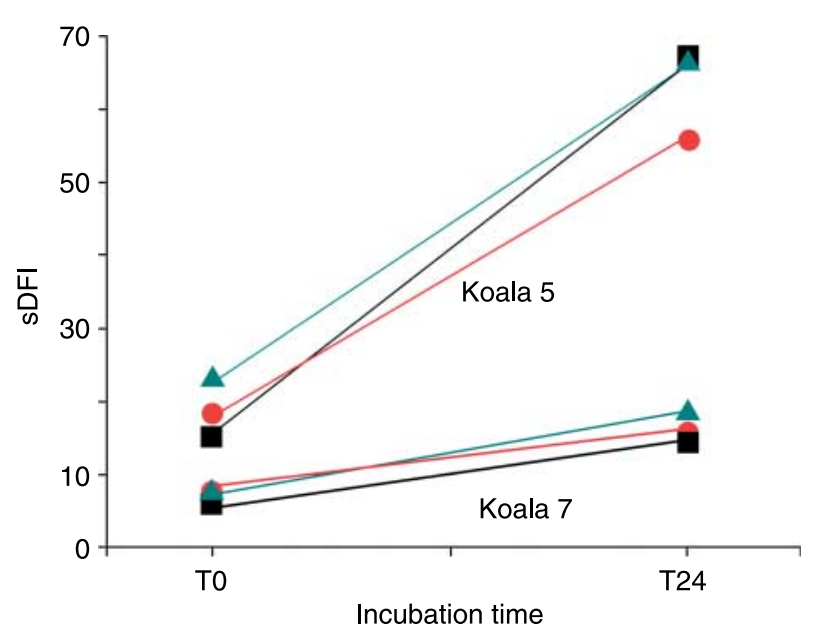

Figure 6 Comparative analysis of sDFI in koala 5 and koala 7 immediately upon thawing (T0) and after $24 \mathrm{~h}$ incubation at $35{ }^{\circ} \mathrm{C}(\mathrm{T} 24)$ using three different methods of sDFI assessment. Black square - SCDt; red circle - neutral comet assay; blue triangle - two-dimensional (neutral + alkaline) comet assay.

relaxation', which presumably renders the spermatozoon unsuitable for fertilisation. We originally proposed that sperm DNA fragmentation could explain a large part of this post-thaw chromatin behaviour (Johnston et al. 2007), but have subsequently demonstrated that this was not the case (Y Zee, C López-Fernández, J Gosálvez, A Gosálbez, WV Holt, CD Allen, V Nicolson, M Pyne \& SD Johnston, unpublished observations) and have shown that the level of DNA in situ nick translation labelling observed in swollen nuclei is very low or non-existent. In the absence of a distinct relationship between DNA fragmentation and chromatin relaxation, as well as from the results of this study, we propose a new hypothesis that chromatin relaxation in koala spermatozoa without DNA fragmentation may be the due to the massive presence of DNA motifs that are highly sensitive to alkaline treatments. These form part of the constitutive structure of the koala sperm DNA molecule and may be important for the packaging of the DNA molecule during spermiogenesis.

The results presented in this study also provide an interesting insight into aspects of DNA status in the koala spermatozoa, both at a structural and functional level. Following the double comet assay, all spermatozoa had either one or two comet tails, demonstrating the pervasiveness of DNA breaks in koala spermatozoa. Spermatozoa with single comet tails produced under alkaline conditions are indicative of the presence of DNA abasic-like residues susceptible to denaturation in the presence of a mild alkali. The pervasiveness of abasic-like residues suggests that constitutive ALSs are part of the structural configuration of the koala sperm nucleus. ALSs are lesions or modifications in the DNA that, with alkaline treatment, can be transformed into single-stranded DNA stretches. The actual location of these ALS in koala sperm nucleus remains to be confirmed, but we do know that they are present in the basal region of the sperm nucleus of the closely related common wombat (Vombatus ursinus; E Cortés Gutierrez, personal communication). The fact that ALS can be found in all koala spermatozoa, even in those with proven fertility (Koala 5), also suggests that these sites are structurally important to the koala spermatozoa, probably for the assembly of chromatin during nuclear condensation associated with spermiogenesis. Sperm ALSs have also been observed in the sperm nucleus of various eutherians such as humans, sheep, pig and mouse (Singh et al. 1989, Cortés-Gutiérrez et al. 2008b), suggesting that these DNA conformations are an important component in the distinctive structure of the sperm chromatin. However, the role they play or their origin is still not completely understood.

According to theories of the origin of DNA fragmentation in spermatozoa, a variety of different lesion types can occur in the DNA; nucleases, either endogenous or exogenous, produce DNA SSB and/or DSB (Agarwal \& Said 2003). Chromatin remodelling during spermiogenesis is believed to necessitate endonuclease (topoisomerase II) activity, which creates nicks that facilitate protamination. These DNA breaks seem to correspond to the presence of DSB (Laberge \& Boissonneault 2005), are not detected at later stages of spermiogenesis and therefore appear to be of a transient nature (Marcon \& Boissonneault 2004). This phenomenon presupposes a DNA repair mechanism that occurs before the completion of the nuclear condensation stage; transition proteins (TNP) and protamines have been known to enhance the ligation of DNA fragments (Sheflin et al. 1991, Lévesque et al. 1998, Caron et al. 2001).

The existence of DNA abasic-like residues in all mature koala spermatozoa may be related to the fact that marsupial P1 protamines lack cysteine residues, which are essential for the formation of disulphide bonds (Temple-Smith 1994). Abnormalities during spermiogenesis could result in an incomplete chromatin maturation process, whereby DNA breaks remain 'unrepaired' giving rise to true DNA damage or producing DNA configurations different to a conventional doublestranded helix that persist in mature spermatozoa (Boissonneault 2002). Interestingly, the presence of transient triplex DNA configurations that possibly exert epigenetic control as protamine replacement takes place was suggested in the elongating spermatids of a grasshopper (Černá et al. 2008). Mice with targeted deletions of Tnp1 or Tnp2 genes or with the disruption of one copy of the gene for either P1 or P2 protamines had an increase in the frequency of spermatozoa with damaged DNA (Cho et al. 2001).

These observations reinforce the idea that, in mature spermatozoa, there exists more than the standard doublestranded and continuous DNA molecular arrangements, and that alternative functional, deleterious or lethal DNA 
configurations may also exist. The existence of an alternative DNA configuration such as that described above would not be lethal even with the possibility of them becoming DNA nicks. This is because most repetitive DNA sequences are non-protein coding; the probability of DNA damage affecting exonic or proteincoded regions would therefore be low (Alvarez 2005, Makhlouf \& Niederberger 2006). This theory has been used as an explanation for the occurrence of viable pregnancies despite high levels of sperm DNA fragmentation. Nevertheless, SSB may trigger an apoptotic pathway after fertilisation or may remain unrepaired or misrepaired, resulting in structural chromosomal aberrations that lead to cell death in the first or subsequent cell cycles, which are independent of the coding or non-coding nature of the DNA region where they are located (van Gent et al. 2001). Hence, the relationship between sperm DNA lesions and fertility is a very complex interaction that needs extensive research.

Another point of interest in this study is the dynamic behaviour of the DNA damage in koalas. The incidence of DSB in both koalas ( $\mathrm{K} 5$ and $\mathrm{K} 7$ ) examined in this study increased when spermatozoa were incubated for $24 \mathrm{~h}$ at $35{ }^{\circ} \mathrm{C}$ following cryopreservation. It is interesting to note that K5, which exhibited a higher incidence of sperm DNA fragmentation immediately upon thawing, also showed a massive increase in sDFI after $24 \mathrm{~h}$, whereas the increase in sDFI of $K 7$ under the same conditions showed only a moderate increase. These results support the idea that sperm DNA fragmentation is a dynamic process, and that relative differences in sperm DNA fragmentation may be better elucidated if spermatozoa are stressed following cryopreservation under the postthaw conditions they would normally experience in the female reproductive tract. The question now is; how is this DNA damage produced within such a relatively short period of time? Reactive oxygen species (ROS) and other radical molecules like those derived from nitric oxide mainly generate DNA SSB and many different DNA base damages (Burney et al. 1999). DNA DSB are generally not expected to be produced directly by ROS, although ROS could elicit DSB through nuclease activity (Macip et al. 2003).

In the case of koala spermatozoa, the trigger for DNA DSB generation seems to be more directly related to enzymatic actions than oxidative stress. This is important in terms of fertility because the most serious or genotoxic forms of DNA damage are DSB. In somatic cells, these are mainly responsible for the production of chromosomal aberrations and the initiation of apoptosis (van Gent et al. 2001). We are not convinced that ROS is the main cause of structural ALS in the koala sperm nuclei as they are present in sperm samples of highly fertile animals. In addition, oxidative DNA damage is typically randomly produced in the DNA, whereas the results of this study have shown that comet tails produced after alkaline treatment in the double comet assay were of a constant length (Fig. 4a-d), suggesting that these sites are homogenously distributed all over the sperm genome.

We are currently exploring the idea that the maximum length of the comet tail represents the distance between two consecutive ALS affected by the alkaline treatment, while the different amounts of DNA recovered in the tails are representative of the number of affected ALS. In fact, the comet tail has also been described as consisting of relaxed loops caused by DNA nicks and that the relative tail intensity is indicative of the number of DNA breaks (Collins et al. 1997). This might explain why the length of the single comet tails observed in this study (Fig. 4a-d) remained constant while the tail density increased, as the length of the comet tail is essentially determined by the length of the relaxed loops. This form of comet corresponded with koala sperm morphotypes 1 and 2 from SCDt (Table 2) and represented spermatozoa with non-fragmented DNA.

Our model of a regular distribution of ALS also coincides with observations of the distribution of ALS in other species, where they do not appear to be randomly distributed throughout the genome, but are mainly concentrated at specific repetitive satellite DNA sequences located in the pericentromeric regions (Cortés-Gutiérrez et al. 2008a, Gosálvez et al. 2009). Also inherent in the idea that sperm DNA damage in the koala is a dynamic process is the possibility that DSB in sperm DNA may be the result or a function of the underlying SSB that exist, not as part of the normal constitutive structure of the DNA, but from ROS or similar apoptotic processes following cryopreservation, prolonged incubation in vitro or exposure to the female reproductive tract. Here, we would define another category of SSB that ultimately predisposes the sperm nucleus to DSB; this category of SSB may in fact be represented by comets that exhibited no DNA migration in neutral conditions, but variable degrees of migration in alkaline conditions.

In conclusion, this study has revealed, by means of neutral and alkaline comet assays, that frozen-thawed koala spermatozoa show evidence of DNA damage ranging from SSB associated with what is proposed to be part of the normal constitutive structure of the chromatin, a more severe category of SSB that might ultimately lead to the production of DSB and sperm nuclei with actual DSB or true DNA fragmentation. The latter category can be further subdivided into those that exhibit obvious migration of DNA fragments under neutral conditions to those with such highly degraded DNA that the fragments have migrated far away from their original core and therefore possess only a small nuclear core with no visible comet tail. This study also represents the first use of the double comet assay for spermatozoa in a species that lacks disulphide bonds in their protamines. This technique has proven to be highly effective for the simultaneous evaluation of both DSB and SSB, and is therefore a more sensitive indicator of DNA 
fragmentation than that of the SCDt or the neutral comet assay. Both the SCDt and comet assays in this study have reinforced the idea that sperm DNA damage in frozenthawed koala spermatozoa is likely to be a dynamic process; we propose that this phenomenon needs to be better acknowledged when making interpretations on the significance of the DNA fragmentation, not only in koala spermatozoa but also in mammalian spermatozoa generally.

\section{Materials and Methods \\ Koala semen collection and preservation}

Semen samples were collected within a period of 3 months (the spring breeding season) by electroejaculation (Johnston et al. 1994) from 22 captive koalas in Southeast Queensland, which were aged between 3 and 5 years and clinically healthy at the time of semen collection. Freshly collected semen samples were diluted $(1: 1)$ with warmed $\left(35^{\circ} \mathrm{C}\right)$ Tris-citrate glucose buffer $\left(35^{\circ} \mathrm{C}\right.$; normal koala body temperature) (TCG: $3.0 \mathrm{~g}$ Tris base (Sigma-Aldrich)), $1.7 \mathrm{~g}$ citric acid (Ajax Finechem, Taren Point, NSW, Australia) and $1.25 \mathrm{~g}$ glucose (Ajax Finechem) made up to $100 \mathrm{ml}$ with Milli-Q Ultrapure water, and adjusted to a $\mathrm{pH}$ of 7.4 and $350 \mathrm{mOsm} / \mathrm{kg}$; (Johnston et al. 2000) in a warmed 1.5-ml Eppendorf microcentrifuge tube (Eppendorf AG, Hamburg, Germany). The extended semen was then allowed to equilibrate in a portable refrigerator $\left(4^{\circ} \mathrm{C}\right)$ while being transported back to the processing laboratory $\sim 2 \mathrm{~h}$ away. Chilled freezing media consisting of TCG and $28 \%$ glycerol were added dropwise to the chilled, extended semen for a final glycerol concentration of $14 \%$. Semen samples were then drawn into chilled $0.25 \mathrm{ml}$ straws (IMV, L'Aigle, France), sealed and frozen in a programmable freezer at $-6{ }^{\circ} \mathrm{C} / \mathrm{min}$ (Model - Freeze Control CL-863, Cryologics Pty Ltd, Mulgrave, VIC, Australia) to $-80^{\circ} \mathrm{C}$. Straws were then removed from the freezer and plunged directly into liquid nitrogen.

Frozen semen from 22 koalas was shipped to the Autonomous University of Madrid (Spain) for further analysis. The SCDt was performed on all 22 koalas and was part of a parallel study investigating the level of SCDt within two captive koala populations (Dreamworld and Currumbin Wildlife Sanctuary) in Southeast Queensland (Y Zee, C López-Fernández, J Gosálvez, WV Holt \& SD Johnston, unpublished observations). From these 22 koalas, two were chosen (Koala 5 and Koala 7) because they showed a consistently high (K5) and a low (K7) level of sperm DNA fragmentation when analysed over a 24-h period at $35^{\circ} \mathrm{C}$. $\mathrm{K} 5$ provided a range of sperm morphotypes that could be further analysed by comet assays, while K7 was as used as a control. These two koalas were sired by the same male and both had sired their own offspring.

\section{Evaluation of basic semen parameters}

Progressive motility was assessed on a phase-contrast microscope fitted with a warm stage set at $35^{\circ} \mathrm{C}$. Motile spermatozoa were those that were moving forward, while those that were moving in circles or with only tail movement were considered as non-motile. Mitochondrial membrane potential (MMP) and plasma membrane integrity (live, dead) were assessed using JC-1 and propidium iodide $(\mathrm{PI})$ respectively in a dual-labelling technique. JC-1 $(2.9 \mu \mathrm{g} / \mathrm{ml}$ or a final concentration of $2 \mu \mathrm{M})$ and PI $(1 \mu \mathrm{l}$ of $2.4 \mathrm{mM}$ stock/100 $\mu \mathrm{l}$ cell suspension) were added to each thawed sample and incubated for $30 \mathrm{~min}$. A Nikon Eclipse E400 microscope equipped with epifluorescent optics (FITC 465-495 excitation filter; DM 505 dichroic mirror; BA 515-555 barrier filter) was used to visualise labelled spermatozoa. Spermatozoa were classified into four categories: 1) live spermatozoa (PInegative) with high MMP (LHMMP); 2) live spermatozoa with low MMP (LLMMP); 3) PI-positive spermatozoa with high MMP; and 4) PI-positive spermatozoa with low MMP. Spermatozoa with high MMP had $50 \%$ or more of their mid-piece stained bright orange and the remaining section of the mid-piece stained green, while spermatozoa with low MMP had $<50 \%$ of their mid-piece stained orange, or the entire mid-piece stained green (Zee et al. 2007). Spermatozoa with an expanded or swollen nucleus were regarded as having relaxed chromatin (Johnston et al. 2006).

\section{Sperm chromatin dispersion test}

To determine the sDFI and to visualise the extent of DNA damage, the SCDt was conducted as described by (Johnston et al. 2007) using a Halomax kit (ChromaCell SL, Madrid, Spain). Thawed semen samples ( $1 \mathrm{~min}$ in $35^{\circ} \mathrm{C}$ waterbath) were diluted in TCG buffer to attain a final sperm concentration of $\sim 10 \times 10^{6}$ spermatozoa per ml. Extended semen $(20 \mu \mathrm{l})$ was added to a vial of low melting agarose that had been melted at $70{ }^{\circ} \mathrm{C}$ for $5 \mathrm{~min}$ and equilibrated to $35^{\circ} \mathrm{C}$ in heat blocks (Thermoline, Brisbane, Australia) and gently mixed. Aliquots $(15 \mu \mathrm{l})$ of the semen-agarose mixture were pipetted onto chilled, pre-treated glass slides provided in the Halomax kit and cover slips $(18 \times 18 \mathrm{~mm})$ placed over this mixture. The slides were then placed on a chilled $\left(4^{\circ} \mathrm{C}\right)$ metallic plate and left to solidify in a refrigerator $\left(4^{\circ} \mathrm{C}\right)$ for $5 \mathrm{~min}$, after which time the cover slips were removed. The slide was then placed horizontally in a plastic tray containing $10 \mu$ of lysing solution (provided in the Halomax kit) for 5 min. The slide was removed from the lysing solution and washed in distilled water for $5 \mathrm{~min}$ and then dehydrated through a series of 2-min ethanol baths (70, 90 and 100\%). GelRed (Biotium, Hayward, CA, USA) was used to visualise the sperm DNA.

\section{Comet assays}

Single-cell gel electrophoreses of sperm DNA (comet assays) were conducted on the semen samples after thawing to identify the presence of SSB and DSB in koala sperm DNA. While the neutral comet assay reveals DSB, the alkaline comet assay permits the detection of SSB. In the current study, a 'twodirectional' or double comet assay was developed for marsupial spermatozoa; in this assay, after the usual lysis, spermatozoa were electrophoresed using a neutral buffer, followed by a second electrophoresis using an alkaline buffer. This experimental approach enabled the simultaneous identification of DSB and SSB in the same spermatozoon.

The procedure for the preparation of microgel slides for both comet assays was similar to that for the SCDt. For the neutral 
comet assay, the microgel slide was treated with the lysis solution used in the SCDt for $5 \mathrm{~min}$ and then removed and placed in $1 \times$ Tris-borate-EDTA (TBE) buffer solution $(0.089 \mathrm{M}$ Tris, $0.089 \mathrm{M}$ boric acid and $0.002 \mathrm{M}$ EDTA) for $15 \mathrm{~min}$. The microgels were then electrophoresed (12 min; $20 \mathrm{~V}$ ) in TBE $(1 \times)$ buffer, during which time DNA fragments resulting from DSB migrate from the nucleus towards the anode. Once electrophoresis was complete, the slide was removed and placed in $0.9 \%$ sodium chloride solution for $2 \mathrm{~min}$, followed by $5 \mathrm{~min}$ in $0.4 \mathrm{M}$ Tris hydrochloride buffer $(\mathrm{pH} 7.5)$ and $2 \mathrm{~min}$ in TBE $(1 \times)$. An ethanol series $(70,90$ and $100 \%$ ) was used to desiccate the microgels.

The microgel slide for the double comet assay was processed through the same procedure as that for the neutral comet assay up to the step where it was placed in $0.9 \%$ sodium chloride for 2 min. Thereafter, the slide was transferred to chilled $\left(4^{\circ} \mathrm{C}\right)$ alkaline solution $(0.03 \mathrm{M}$ sodium hydroxide and $1 \mathrm{M}$ sodium chloride; $\mathrm{pH} 12.5)$ for $2 \mathrm{~min} 30 \mathrm{~s}$. The slide was then electrophoresed $(20 \mathrm{~V} ; 4 \mathrm{~min})$ in alkaline solution at room temperature, but with the slide repositioned $90^{\circ}$ clockwise to its original position in the first electrophoresis. This step, performed under alkaline conditions, resulted in the release of DNA fragments caused by SSB to migrate towards the anode during application of the electrical current while leaving behind DNA fragments from DSB in their original positions. Subsequently, the slide was placed in Tris hydrochloride buffer for $5 \mathrm{~min}$ followed by $2 \mathrm{~min}$ in TBE $(1 \times)$ buffer and the microgels desiccated in a series of ethanol baths. Sperm DNA in both assays was visualised using GelRed (Biotium).

\section{Image processing and analysis}

All images were captured using a Leica DMA microscope with a 'XYZ' motorised holder (Leica Microsystems, Barcelona, Spain). Black and white images were captured using 12-bit format with a CCD (Leica DFC350 FX, Leica Microsystems). Images were stored as Tiff black and white 12-bit format. Image analysis was performed to compare the fluorescence intensities. For this purpose, a fixed exposure time was pre-selected for all exposures. In this case, a second capture exposure was used for each spermatozoon after a previous irradiation of each spermatozoon for $5 \mathrm{~s}$ to homogenise fluorochrome fading. Each image was converted to 8 bits after homogeneous subtraction of the null information range in each image. Densitometric profiles were constructed using ImageJ software (NIH Image, Bethesda, Maryland, USA). To enhance image contrast for visualisation effect, 8-bit images were transformed to 24-bit RGB images, pseudo-coloured and filtered using a common electronic filtering series consisting of a 'find edges filter' followed by an 'invert filter' and automatic level adjustment. All these steps were performed using Adobe Photoshop CS3 (Adobe Systems Incorporated).

\section{Declaration of interest}

The authors declare that there is no conflict of interest that could be perceived as prejudicing the impartiality of the research reported.

\section{Funding}

This study was financially supported by the Australian Research Council Linkage Grant Scheme (LP0455785) and the Spanish Ministerio de Educación y Ciencia (BFU2007-66340).

\section{References}

Agarwal A \& Said TM 2003 Role of sperm chromatin abnormalities and DNA damage in male infertility. Human Reproduction Update 9 331-345.

Alvarez JG 2005 The predictive value of sperm chromatin structure assay. Human Reproduction 20 2365-2367.

Aravindan GR, Bjordahl J, Jost LK \& Evenson DP 1997 Susceptibility of human sperm to in situ DNA denaturation is strongly correlated with DNA strand breaks identified by single-cell electrophoresis. Experimental Cell Research 236 231-237.

Boissonneault G 2002 Chromatin remodeling during spermiogenesis: a possible role for the transition proteins in DNA strand break repair. FEBS Letters 514 111-114.

Burney S, Niles JC, Dedon PC \& Tannenbaum SR 1999 DNA damage in deoxynucleosides and oligonucleotides treated with peroxynitrite. Chemical Research in Toxicology 12 513-520.

Caron N, Veilleux S \& Boissonneault G 2001 Stimulation of DNA repair by the spermatidal TP1 protein. Molecular Reproduction and Development 58 437-443.

Černá A, López-Fernández C, Fernández J, Moreno Díaz de la Espina S, de la Torre C \& Gosálvez J 2008 Triplex configuration in the nick-free DNAs that constitute the chromosomal scaffolds in grasshopper spermatids. Chromosoma 117 15-24.

Cho C, Willis WD, Goulding EH, Jung-Ha H, Choi Y-C, Hecht NB \& Eddy EM 2001 Haploinsufficiency of protamine-1 or -2 causes infertility in mice. Nature Genetics 28 82-86.

Chohan KR, Griffin JT, Lafromboise M, De Jonge CJ \& Carrell DT 2006 Comparison of chromatin assays for DNA fragmentation evaluation in human sperm. Journal of Andrology 27 53-59.

Collins AR, Dobson VL, Duinská M, Kennedy G \& Stetina R 1997 The comet assay: what can it really tell us? Mutation Research/Fundamental and Molecular Mechanisms of Mutagenesis 375 183-193.

Cortés-Gutiérrez EI, Crespo F, Gosálvez A, Dávila-Rodríguez MI, LópezFernández C \& Gosálvez J 2008a DNA fragmentation in frozen sperm of Equus asinus: Zamorano-Leonés, a breed at risk of extinction. Theriogenology 69 1022-1032.

Cortés-Gutiérrez EI, Dávila-Rodríguez MI, López-Fernández C, Fernández JL \& Gosálvez J $2008 b$ Alkali-labile sites in sperm cells from Sus and Ovis species. International Journal of Andrology $\mathbf{3 1}$ 354-363.

Enciso M, Muriel L, Fernandez JL, Goyanes V, Segrelles E, Marcos M, Montejo JM, Ardoy M, Pacheco A \& Gosalvez J 2006 Infertile men with varicocele show a high relative proportion of sperm cells with intense nuclear damage level, evidenced by the sperm chromatin dispersion test. Journal of Andrology 27 106-111.

Evenson DP, Larson KL \& Jost LK 2002 Sperm chromatin structure assay: its clinical use for detecting sperm DNA fragmentation in male infertility and comparisons with other techniques. Journal of Andrology 23 25-43.

Fernandez JL, Muriel L, Rivero MT, Goyanes V, Vazquez R \& Alvarez JG 2003 The sperm chromatin dispersion test: a simple method for the determination of sperm DNA fragmentation. Journal of Andrology $\mathbf{2 4}$ 59-66.

Fifis T, Cooper DW \& Hill RJ 1990 Characterization of the protamines of the Tammar wallaby (Macropus eugenii). Comparative Biochemistry and Physiology. Part B, Biochemistry \& Molecular Biology 95 571-575.

van Gent DC, Hoeijmakers JHJ \& Kanaar R 2001 Chromosomal stability and the DNA double-stranded break connection. Nature Reviews. Genetics 2 196-206.

Gorczyca W, Traganos F, Jesionowska H \& Darzynkiewicz Z 1993 Presence of DNA strand breaks and increased sensitivity of DNA in situ to denaturation in abnormal human sperm cells: analogy to apoptosis of somatic cells. Experimental Cell Research 207 202-205. 
Gosálvez J, Fernández JL, Goyanes V \& López-Fernández C 2006 Análisis de la fragmentación del ADN en espermatozoides mediante el test de dispersión de la cromatina (SCD). Biotechnology 1 38-51.

Gosálvez J, Cortés-Gutiérrez EI, Dávila-Rodríguez MI, Fernández JL, López-Fernández C \& Johnston SD 2009 Mapping alkali-labile sites in mammalian spermatozoa. In Animal Reproduction: New Research Developments [in press]. Hauppauage, New York: Nova Science Publishers, Inc.

Johnston SD, McGowan MR, Carrick FN, Cameron RDA \& Tribe A 1994 Seminal characteristics and spermatozoal morphology of captive Queensland koalas (Phascolarctos cinereus). Theriogenology 42 501-511.

Johnston SD, McGowan MR, Phillips NJ \& O'Callaghan P 2000 Optimal physicochemical conditions for the manipulation and short-term preservation of koala (Phascolarctos cinereus) spermatozoa. Journal of Reproduction and Fertility 118 273-281.

Johnston SD, MacCallum C, Blyde D, McClean R, Lisle A \& Holt WV 2006 An investigation into the similarities and differences governing the cryopreservation success of koala (Phascolarctos cinereus: Goldfuss) and common wombat (Vombatus ursinus: Shaw) spermatozoa. Cryobiology $53218-228$.

Johnston SD, Lopez-Fernandez C, Gosalbez A, Zee Y, Holt WV, Allen C \& Gosalvez J 2007 The relationship between sperm morphology and chromatin integrity in the koala (Phascolarctos cinereus) as assessed by the sperm chromatin dispersion test (SCDt). Journal of Andrology $\mathbf{2 8}$ 891-899.

Laberge R-M \& Boissonneault G 2005 On the nature and origin of DNA strand breaks in elongating spermatids. Biology of Reproduction 73 289-296.

Lévesque D, Veilleux S, Caron N \& Boissonneault G 1998 Architectural DNA-binding properties of the spermatidal transition proteins 1 and 2 . Biochemical and Biophysical Research Communications 252 602-609.

Macip S, Igarashi M, Berggren P, Yu J, Lee SW \& Aaronson SA 2003 Influence of induced reactive oxygen species in p53-mediated cell fate decisions. Molecular and Cellular Biology 23 8576-8585.

Makhlouf AA \& Niederberger C 2006 DNA integrity tests in clinical practice: it is not a simple matter of black and white (or red and green). Journal of Andrology 27 316-323.

Marcon L \& Boissonneault G 2004 Transient DNA strand breaks during mouse and human spermiogenesis:new insights in stage specificity and link to chromatin remodeling. Biology of Reproduction 70 910-918.

McPherson SMG \& Longo FJ 1993 Nicking of rat spermatid and spermatozoa DNA: possible involvement of DNA topoisomerase II. Developmental Biology 158 122-130.
Muriel L, Garrido N, Fernández JL, Remohí J, Pellicer A, de los Santos MJ \& Meseguer M 2006 Value of the sperm deoxyribonucleic acid fragmentation level, as measured by the sperm chromatin dispersion test, in the outcome of in vitro fertilization and intracytoplasmic sperm injection. Fertility and Sterility 85 371-383.

Ostling O \& Johanson KJ 1984 Microelectrophoretic study of radiationinduced DNA damages in individual mammalian cells. Biochemical and Biophysical Research Communications 123 291-298.

Sheflin LG, Fucile NW \& Spaulding SW 1991 HMG14 and protamine enhance ligation of linear DNA to form linear multimers: phosphorylation of HMG 14 at Ser 20 specifically inhibits intermolecular DNA ligation. Biochemical and Biophysical Research Communications 174 660-666.

Singh NP, Danner DB, Tice RR, McCoy MT, Collins GD \& Schneider EL 1989 Abundant alkali-sensitive sites in DNA of human and mouse sperm. Experimental Cell Research 184 461-470.

Temple-Smith PD 1994 Comparative structure and function of marsupial spermatozoa. Reproduction, Fertility, and Development 6 421-435.

Tice RR, Agurell E, Anderson D, Burlinson B, Hartmann A, Kobayashi H, Miyamae Y, Rojas E, Ryu JC \& Sasaki YF 2000 Single cell gel/comet assay: guidelines for in vitro and in vivo genetic toxicology testing. Environmental and Molecular Mutagenesis 35 206-221.

Zee YP, Holt WV, Allen CD, Nicolson V, Burridge M, Lisle A, Carrick FN \& Johnston SD 2007 Effects of cryopreservation on mitochondrial function and heterogeneity, lipid raft stability and phosphatidylserine translocation in koala (Phascolarctos cinereus) spermatozoa. Reproduction, Fertility, and Development 19 850-860.

Zee YP, Holt WV, Gosálvez J, Allen CD, Nicolson V, Pyne M, Burridge M, Carrick FN \& Johnston SD 2008 Dimethylacetamide can be used as an alternative to glycerol for the successful cryopreservation of koala (Phascolarctos cinereus) spermatozoa. Reproduction, Fertility, and Development 20 724-733.

Zini A, Kamal K, Phang D, Willis J \& Jarvi K 2001 Biologic variability of sperm DNA denaturation in infertile men. Urology 58 258-261.

Received 25 January 2009

First decision 3 March 2009

Revised manuscript received 12 April 2009

Accepted 3 June 2009 